\title{
High expression of thymidine phosphorylase in basal-like breast cancers: Stromal expression in EGFR- and/or CK5/6-positive breast cancers
}

\author{
SHINOBU UMEMURA ${ }^{1}$, MASATOSHI SHIRANE $^{3}$, SUSUMU TAKEKOSHI ${ }^{1}$, \\ YUTAKA TOKUDA $^{2}$, KAZUSHIGE MORI $^{3}$ and ROBERT Y. OSAMURA ${ }^{1}$ \\ Departments of ${ }^{1}$ Pathology and, ${ }^{2}$ Surgery, Tokai University School of Medicine, Kanagawa 259-1193; \\ ${ }^{3}$ Product Research Department, Chugai Pharmaceutical Co. Ltd., Kanagawa 247-8530, Japan
}

Received October 15, 2009; Accepted December 29, 2009

DOI: 10.3892/ol_00000046

\begin{abstract}
Expression of the estrogen receptor (ER), the progesterone receptor $(\mathrm{PgR})$ or the human epidermal growth factor receptor-2 (HER2) in tumors is a good prognostic marker for breast cancer patients. However, approximately $15-20 \%$ of breast cancer patients have triple-negative breast cancer (TNBC; negative for ER, PgR and HER2), and efficient therapeutic modalities for these patients are under investigation. We focused on thymidine phosphorylase (TP), an enzyme metabolizing 5'-DFUR, an intermediate of capecitabine, to 5-fluorouracil in order to investigate the application of well-known therapeutics for TNBC. Results of a gene expression analysis showed that TP expression in TNBC and basal-like breast cancer (BLBC) was higher than that of other subtypes. Immunohistochemically, the high expression of TP in TNBC and BLBC reflected expression in stromal but not tumor cells. Notably, a high TP expression was observed in the stromal cells of EGFR- and/or CK5/6-positive breast tumors. Our present results showing a high expression of TP in BLBC indicate that capecitabine-based chemotherapy would be of benefit for patients with TNBC.
\end{abstract}

\section{Introduction}

Treatment strategies for breast cancers have been successfully designed in an integrated manner with biological molecules including the estrogen receptor (ER), the progesterone receptor $(\mathrm{PgR})$ and the human epidermal growth factor receptor-2 (HER2) (1). Endocrine therapies, such as tamoxifen or letrozole, and the HER2 antibody (trastuzumab) are used clinically

Correspondence to: Dr Masatoshi Shirane, Product Research Department, Chugai Pharmaceutical Co. Ltd., 200 Kajiwara, Kamakura, Kanagawa 247-8530, Japan

E-mail: shiranemst@chugai-pharm.co.jp

Key words: thymidine phosphorylase, estrogen receptor, human epidermal growth factor receptor-2, basal-like breast tumor for hormonal receptor (HR)- and HER2-positive patients, respectively, and have been found to prolong the survival of breast cancer patients (2). On the other hand, approximately $15-20 \%$ of breast cancer patients who have triple-negative breast cancer (TNBC; ER-, PgR- and HER2-negative) are generally subjected to chemotherapeutic agents. Sørlie et al showed that breast cancer can be clustered into four subtypes: luminal (A,B), HER2-positive, basal-like and normal breastlike cancer. The findings were based on a hierarchical clustering study of gene expression (3-5). The basal-like subtype is one of the categories for which therapeutic strategies are being reconsidered, and many drugs have been proposed as candidates. DNA-damaging drugs including platinum or anthracyclines, DNA-repairing inhibitors including polyAribose 1 (PARP1), and targeted drugs for epidermal growth factor receptor (EGFR) are expected to have benefits for this group. The basal-like subtype was originally a genotypic concept. However, studies have increasingly defined basal-like breast cancer (BLBC) to be a type of breast cancer with the immunophenotype of TNBC and positive for EGFR and/or cytokeratin (CK)5/6 expression (6). TNBC expresses a basal phenotype in $56 \%$ of cases compared with non-TNBC (11.5\%) (7). Thus, TNBC and BLBC are not identical but closely related, and both are associated with poor clinical outcome and lack the benefit of a targeted systemic therapy.

Capecitabine is a widely used chemotherapeutic agent for breast cancer patients (8). Capecitabine was designed as a prodrug which is selectively converted to 5-fluorouracil (5-FU) by thymidine phosphorylase (TP) in tumors (9). TP was first described as an enzyme responsible for nucleoside metabolism, but was later found to be identical to the enzyme extracted from human platelets known as plateletderived endothelial cell growth factor (PD-ECGF) $(10,11)$ and was found to be involved in anti-apoptotic activity and angiogenesis (12). The efficacy of capecitabine may correlate to TP expression in the tumor (13). Some case reports have indicated that capecitabine and docetaxel combination therapy is useful for the treatment of TNBC $(14,15)$. However, it is not well documented whether or not TP expression and the intrinsic subtype of breast cancer are related. Hence, we focused on TP expression in tumor specimens obtained from 
Table I. Clinicopathological features and immunohistochemical phenotype of the breast cancers examined.

\begin{tabular}{|c|c|c|c|c|}
\hline & $\begin{array}{c}\text { ER-positive/ } \\
\text { HER2-negative }\end{array}$ & $\begin{array}{l}\text { ER-positive/ } \\
\text { HER2-positive }\end{array}$ & $\begin{array}{l}\text { ER-negative/ } \\
\text { HER2-positive }\end{array}$ & $\begin{array}{c}\text { ER-negative/ } \\
\text { HER2-negative }\end{array}$ \\
\hline \multicolumn{5}{|l|}{ Tumor size } \\
\hline $\mathrm{T} 1$ & 7 & 3 & 4 & 4 \\
\hline $\mathrm{T} 2$ & 2 & 6 & 2 & 4 \\
\hline $\mathrm{T} 3$ & 1 & 1 & 3 & 2 \\
\hline \multicolumn{5}{|c|}{ Lymph node metastasis } \\
\hline Present & 4 & 4 & 6 & 5 \\
\hline Absent & 6 & 6 & 4 & 5 \\
\hline \multicolumn{5}{|l|}{ Histological grade } \\
\hline 1 & 5 & 0 & 1 & 0 \\
\hline 2 & 5 & 8 & 5 & 4 \\
\hline 3 & 0 & 2 & 4 & 6 \\
\hline \multicolumn{5}{|c|}{ Immunohistochemical phenotype } \\
\hline PgR-positive & 10 & 7 & 0 & 0 \\
\hline EGFR-positive & 0 & 2 & 7 & 7 \\
\hline CK5/6-positive & 1 & 1 & 4 & 8 \\
\hline \multicolumn{5}{|l|}{ TP IHC score } \\
\hline Cancer cell & 1.3 & 1.1 & 1.5 & 1.4 \\
\hline Stroma & 1.4 & 1.7 & 1.9 & 2.0 \\
\hline Total & 2.7 & 2.9 & 3.4 & 3.4 \\
\hline
\end{tabular}

ER, estrogen receptor; PgR, progesterone receptor; HER2, human epidermal growth factor receptor-2; EGFR, epidermal growth factor receptor; CK, cytokeratin; TP, thymidine phosphorylase; IHC, immunohistochemistry.

breast cancer patients with differentially expressed ER/HER2 status in relation to TNBC and BLBC.

\section{Patients and methods}

Patients and tumor specimens. We serially collected 40 tumor specimens consisting of 10 samples in each of the four groups defined by the immunohistochemical expression of ER and HER2: ER-positive/HER2-positive, ER-positive/HER2-negative, ER-negative/HER2-positive and ER-negative/HER2-negative, and analyzed the specimens using an oligonucleotide microarray. A total of 40 tumor tissues including 39 invasive ductal carcinomas and 1 ductal carcinoma in situ were surgically obtained from breast cancer patients following informed consent. This experiment was approved by the ethics committees of both Tokai University and Chugai Pharmaceutical Co. Ltd. The clinicopathological features are shown in Table I. Specimens were resected from the main tumor mass, avoiding areas with massive necrosis and areas intermingling with non-neoplastic breast tissue. Tumor tissue samples were divided into two specimens; one was snap-frozen in liquid nitrogen and stored at $-80^{\circ} \mathrm{C}$ for gene expression analysis and the other was fixed in $10 \%$ formalin within $48 \mathrm{~h}$ and embedded in paraffin for immunohistochemical analysis. For clinical diagnosis, ER was stained by an automated machine for immunohistochemistry (IHC) (Benchmark; Ventana Japan, Yokohama, Japan), and HER2 was stained using a HER2 kit (Dako HercepTest;
DakoCytomation, Carpinteria, CA, USA) according to the manufacturer's protocols. The ER-IHC results were interpreted as positive when $>10 \%$ of the cancer cells immunoreacted. HER2-IHC was assigned scores according to the following ranking: $3+$ (positive), $2+$ (equivocal) and 0 or $1+$ (negative). HER2 was interpreted as positive when IHC $3+$ or the HER2/CEP17 signal ratio was $\geq 2.2$ from fluorescence in situ hybridization (FISH) for IHC $2+$ tumors. None of the patients had received any pre-operative adjuvant hormone treatment or chemotherapy.

Evaluation of gene expression. Details of the oligonucleotide microarray analysis were as previously described (16). Briefly, total RNA was extracted from the frozen tissues with Sepazol-I (Wako, Osaka, Japan). The RNA was reversetranscribed to cDNA using T7-(dT)24 primer. Biotin-labeled cRNA was synthesized from cDNA using a MegaScript In Vitro Transcript Kit (Ambion, Austin, TX, USA), and then hybridized to human U95Av2 GeneChip ${ }^{\circledR}$ (Affymetrix, Santa Clara, CA, USA). The hybridized oligonucleotide microarrays were scanned using a confocal scanner (Affymetrix) and analyzed using Affymetrix software (LIMS 5.0). The signal intensity of TP was expressed as a logarithmic value.

Immunohistochemical analysis for thymidine phosphorylase and the basal phenotype. Specimen sections $(4 \mu \mathrm{m})$ were mounted on silane-coated glass slides, deparaffinized in graded xylene and dehydrated in ethanol. After the inhibition of 
intrinsic peroxidase by immersion in methanol with $0.3 \% \mathrm{H}_{2} \mathrm{O}_{2}$ for $30 \mathrm{~min}$, antigen retrieval pretreatment was carried out by twice heating in a microwave oven for $5 \mathrm{~min}(500 \mathrm{~W})$ in $0.01 \mathrm{M}$ citrate buffer, $\mathrm{pH}$ 6.0. Anti-human mouse TP monoclonal antibody (1C6-203) (16), anti-human EGFR antibody (clone 2-18C9, EGFR pharmDx Kit; DakoCytomation), anti-human CK5/6 antibody (clone D5/16B4; DakoCytomation) and antihuman PgR were used in this study. After incubation of the TP primary antibody, mouse IgG (ICN Pharmaceuticals, Inc., Costa Mesa, CA, USA) was soaked in $0.1 \mathrm{M}$ phosphate buffer ( $\mathrm{pH}$ 7.4) containing 3\% blocking serum and $0.3 \%$ Triton X-100 and incubated at $4^{\circ} \mathrm{C}$ overnight. The slides were washed with PBS, incubated with biotin-labeled secondary antibody and detected using a Vectastain Elite ABC Kit (Vector Laboratories, Inc., Burlingame, CA, USA). The levels of immunostained TP in the tumor or stromal cells were independently assigned a score $(0,1,2$, or 3 , from lowest to highest) based on a previous report (18). Total TP expression was estimated from the sum of the scores observed in the tumor and stromal cells. PgR was stained by an automated machine for IHC, and EGFR was stained according to the manufacturer's protocols. Breast cancers that were negative for the expression of ER, PgR and HER2 were determined to be TNBCs, and breast cancer samples lacking an expression of ER, PgR and HER2 but expressing EGFR and/or CK5/6 were considered to be BLBCs (6).

Statistics. Statistical analysis was performed using StatView 5.0 (Hulinks Inc.) on a Windows PC. $\mathrm{P}<0.05$ was considered to be of statistical significance.

\section{Results}

Thymidine phosphorylase mRNA expression. As shown in Fig. 1A, TP expression (array signal intensity of logarithm, 2.8184; 95\% CI 2.7071-2.8833) was significantly higher in the tumors than that in adjacent normal tissues (2.4260; 95\% CI 2.1112-2.5975) ( $\mathrm{p}=0.0061$, Mann-Whitney U test). To investigate the relationship between TP expression and ER or HER2 status, the expression levels of TP were compared between ER-positive and ER-negative (Fig. 1B), and between HER2-positive and HER2-negative (Fig. 1C) breast tumors. ER-negative breast tumors $(2.9270 ; 95 \%$ CI 2.8352-3.0171) showed a significantly higher TP expression than ER-positive breast tumors $(2.7529$; 95\% CI 2.5370-2.7914) ( $\mathrm{p}=0.0035$, Mann-Whitney U test), whereas there was no significant difference in TP expression between the HER2-positive and HER2-negative breast tumors $(\mathrm{p}=0.2448$, Mann-Whitney $\mathrm{U}$ test). To further investigate, we compared TP expression between the ER-positive/HER2-positive, ER-positive/HER2negative, ER-negative/HER2-positive and the ER-negative/ HER2-negative tumor subtypes. In the multivariate analysis (Fig. 1D), TP expression in ER-negative/HER2-negative tumors (3.0641; 95\% CI 2.8886-3.1392) was higher than that in ER-positive/HER2-positive (2.7179; 95\% CI 2.3667-2.8321) and ER-positive/HER2-negative tumors (2.7529; 95\% CI 2.6435-2.8137). In addition to the ER and HER2 status, the expression of PgR, EGFR and CK5/6 is summarized in Table I. The ER-negative/HER2-negative tumors were also negative for PgR and were considered to be TNBC. Moreover,
A
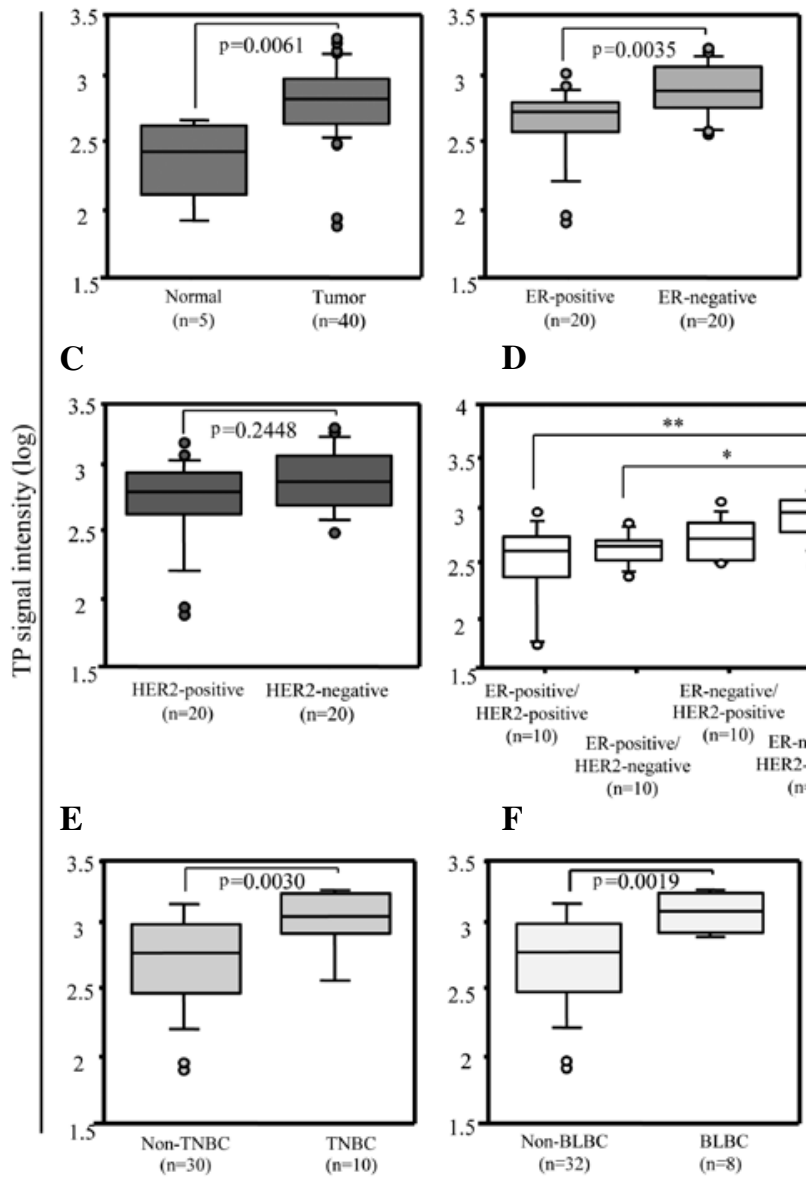

Figure 1. Box plot of TP mRNA expression in breast tumors. Plots were scaled by dividing each median value. TP mRNA expression in (A) breast tumor and adjacent normal tissues, (B) ER-positive and ER-negative breast tumors, and (C) HER2-positive and HER2-negative breast tumors. (D) Multivariate analysis of TP expression in ER-positive/HER2-positive, ER-positive/HER2-negative, ER-negative/HER2-positive and ER-negative/ HER2-negative breast tumors. TP mRNA expression in (E) triple-negative breast cancer (ER/PgR/HER2-negative, TNBC) and non-TNBC, $(\mathrm{F})$ basallike breast cancer (BLBC) and non-BLBC. Statistical analysis was performed by the Mann-Whitney U test (A-C, E and F) or by one-way analysis of variance followed by Dunnett's t-test (D). $\mathrm{P}<0.05$ was considered statistically significant $\left({ }^{*} \mathrm{p}<0.05,{ }^{* *} \mathrm{p}<0.01\right)$.

8/10 of the ER-negative/HER2-negative tumors were BLBC, expressing EGFR and/or CK5/6. We then compared TP expression of TNBC or BLBC to that of the other tumor groups. TP expression was higher in TNBC $(\mathrm{p}=0.0030$, Mann-Whitney U test) (Fig. 1E) and BLBC ( $\mathrm{p}=0.0019$, MannWhitney $U$ test) (Fig. 1F) than in non-TNBC and non-BLBC tumors, respectively.

Immunohistochemical analysis of thymidine phosphorylase. In addition to comparing the mRNA levels, we investigated TP expression immunohistochemically in breast cancer tissues. Typical patterns of TP expression in cancers are shown in Fig. 2A including no expression (top left), expression in stroma (top right), expression in cancer cells (bottom left), and expression in both stroma and cancer cells (bottom right). The proportion of TP expression in cancer cells and stroma varied from tumor to tumor in the four groups of breast 
A

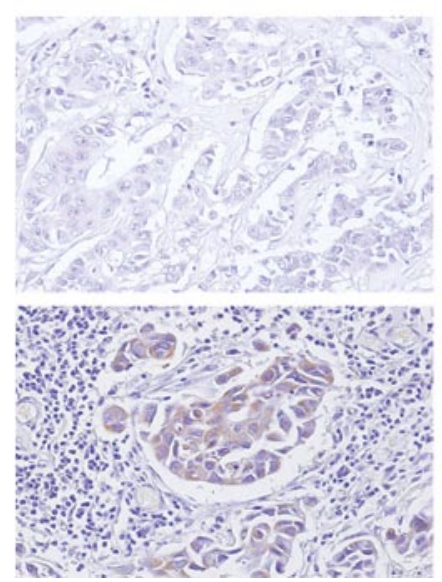

A

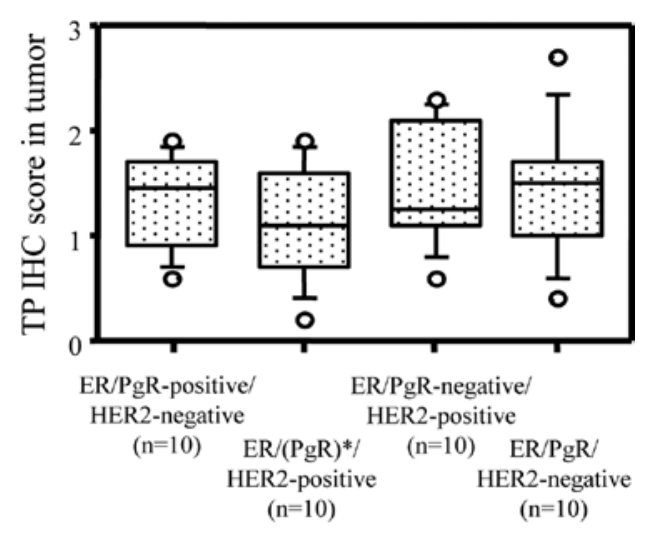

$\mathbf{B}$

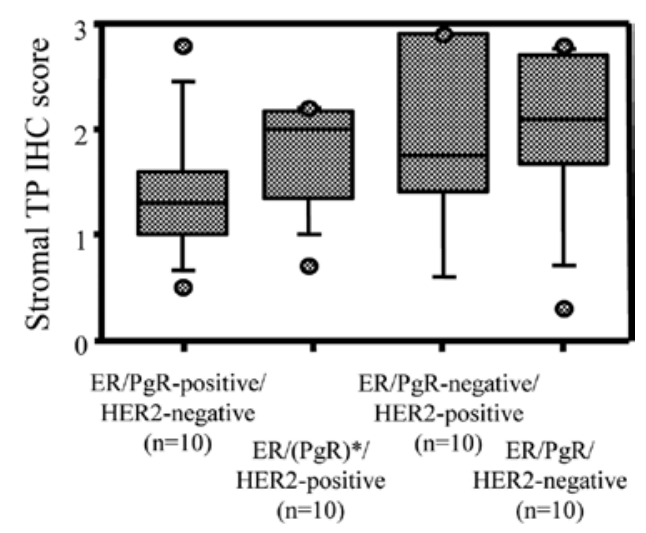

Figure 3. Box plot for total (A) and stromal (B) TP immunohistochemical scores in ER/PgR-positive/HER2-negative, ER/(PgR)*/HER2-positive, ER/ PgR-negative/HER2-positive and ER/PgR/HER2-negative breast tumors $(* 7 / 10$ tumors were positive for $\mathrm{PgR})$. Plots were scaled by dividing each median value.
A

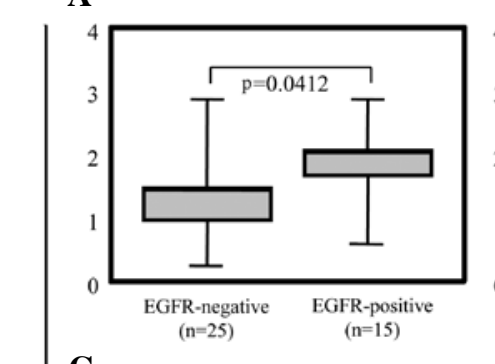

C

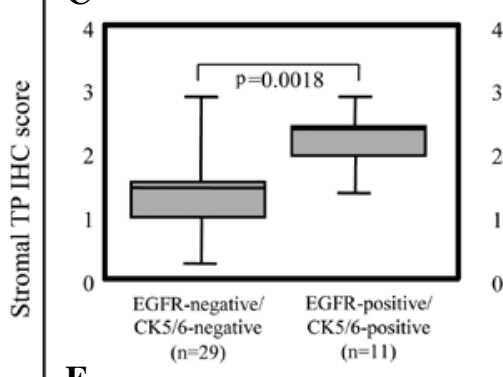

$\mathbf{E}$

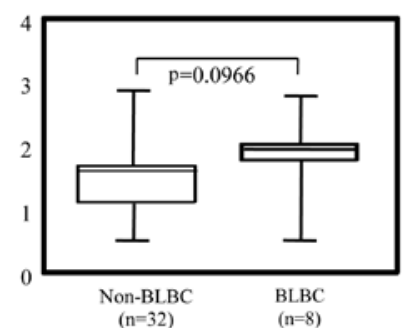

B

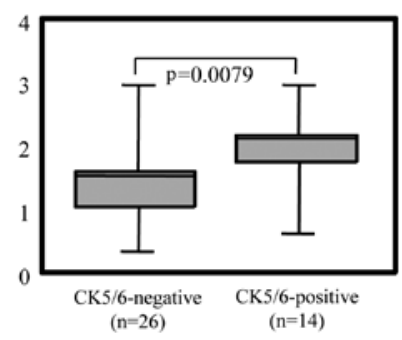

D

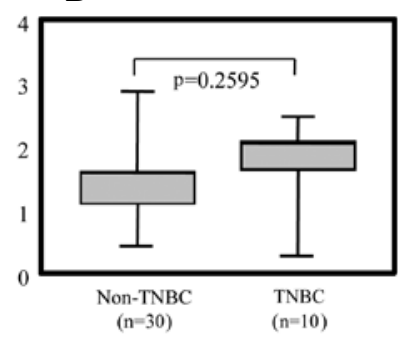

tumors. Subsequently, the tumor samples were evaluated according to previously reported scores (13). Among the 40 specimens, total immunohistochemical TP staining scores weakly but significantly correlated to mRNA expression $(\mathrm{r}=0.34, \mathrm{p}=0.034)$ (Table I, Fig. 2B). Although total TP expression scores showed no differences among the ER/ PgR/HER2-positive, ER/(PgR)-positive/HER2-negative, ER/ PgR-negative/HER2-positive and ER/PgR/HER2-negative breast tumors (Fig. 3A), TP expression in the stromal cells tended to be higher in ER-negative breast cancer than in ER-positive breast cancer (Fig. 3B).

We further analyzed the correlation between TP expression in the stromal cells and basal phenotypes of breast cancer. TP expression in the stromal cells of the EGFR- and CK5/6positive breast tumors was significantly higher than that in each negative group (Fig. 4A and B). Notably, both EGFRand CK5/6-positive breast tumors showed extensively higher $\mathrm{TP}$ expression in the stromal cells (Fig. 4C). TP expression in the stromal cells demonstrated a tendency related to BLBC ( $\mathrm{p}=0.0979$, Mann-Whitney $\mathrm{U}$ test) (Fig. 4E), even though

Figure 4. Box plot of immunohistochemical scores for TP expression of stromal cells in EGFR-positive (A), CK5/6-positive (B), EGFR-positive/ CK5/6-positive breast tumors (C), triple-negative breast cancer (D) and basallike breast cancer (E). Plots were scaled by dividing each median value. 
TNBC showed no tendency with TP expression in stromal cells (Fig. 4D). Taken together, the high level of expression of TP in the stromal cells correlated with ER-negative breast cancers expressing CK5/6 and EGFR.

\section{Discussion}

The present study demonstrated that the expression of the TP gene was higher in TNBC and BLBC. Immunohistochemical analysis results showed that a higher TP expression in the stroma significantly correlated with ER-negative breast cancers expressing EGFR and CK5/6. Intracellular signaling via ER or HER2 is considered essential for tumor growth and/or anti-apoptosis, and there are therapeutic strategies to inhibit these receptors such as tamoxifen, letrozol and trastuzumab. However, in ER-negative/HER2-negative breast tumors, the transmitted signals that result in tumorigenesis and anti-apoptosis remain unclear. TNBC and BLBC share clinical features such as a poor patient outcome. Moreover, they lack the benefit of a targeted therapy $(3-5,19)$. It is widely accepted that TNBC is heterogeneous and includes BLBC, as a significant subgroup, as well as non-basal phenotypes. Among the specific markers for BLBC such as EGFR, CK5/6, BRCA1, c-kit and p53 mutation (3-5), EGFR or c-kit may be candidate treatment targets. Although EGFR overexpression has been observed in approximately half (57\%) of BLBCs (19), at present insufficient evidence exists regarding a treatment targeting EGFR in BLBC patients $(20,21)$. TP exerts tumorigenic, anti-apoptotic and angiogenic functions (12) and, considering the results of the present study, TP is a reasonable target for patients with BLBC.

Our results contributed to the elucidation of the relationship between cancer cells and stroma. The expression level of the TP gene in tumor subtypes correlated with TP IHC scores in the stroma, which were higher. Stromal tissue is presumed to be a significant source of TP gene expression throughout the entire tumor. Since the stromal compartment has been reported to be associated with breast tumor initiation and progression (22), many investigators recently focused on this relationship. Stromal gene expression profiles can predict clinical outcome for patients with breast tumors (23). Stromal cells play an important role in breast tumors, and the microenvironment that includes the tumor and stromal cells needs to be considered when evaluating drug sensitivity to breast tumors. With regard to TP expression, it was demonstrated that human TP cDNA transfection in colorectal (24) and non-small lung cancer cells (25) increased sensitivity to 5'-DFUR and induced a bystander effect towards co-cultured parental cells with increased 5'-DFUR sensitivity. Therefore, it is theoretically expected that stromal TP would induce a bystander effect towards adjacent tumor cells when treated with capecitabine. Since capecitabine was designed as a pro-drug that is selectively converted to 5-FU by TP in tumor tissue (9), it is expected to be more efficiently converted to 5-FU in a tumor microenvironment with a higher expression level of TP even though it is expressed in the stroma.

It remains unclear why the expression of TP in stromal and cancer cells is higher in ER-negative expressing EGFR- and CK5/6-positive breast cancers. TP expression is induced by hypoxia and several cytokines such as tumor necrosis factor $\alpha$, interleukin 1, or interferon $\gamma$ (26). One possible mechanism is that ER-negative and EGFR/CK5/6-positive cancer cells secrete cytokines that may cause the stromal cells to induce a high TP in the stroma. Notably, there were no significant differences between the TP IHC scores for TNBC/non-TNBC and $\mathrm{BLBC} /$ non-BLBC, possibly because there is no correlation between TP expression and HER2 that offsets the difference between TNBC/non-TNBC and BLBC/non-BLBC. It is noteworthy that EGFR- and CK5/6-positive cancer cells are more intimately related to stromal TP expression than HER2positive breast cancers.

The hypoxic mechanism of TP induction is one of the explanations for the efficacy of capecitabine in tumors after treatment with other anticancer drugs (12). In preclinical studies, capecitabine showed at least an additive effect in human breast cancer xenograft models in combination with trastuzumab (23) and endocrine therapies (28). In human cancer tissues of various organs, TP expression was demonstrated to be significantly higher in tumors than in adjacent normal tissues, regardless of the ER or HER2 status [Kataoka et al, Cancer Res 69 (Suppl 2): abs. 2012, 2009]. In the clinical setting, capecitabine is often administered in combination with other anticancer drugs for breast cancers, and combination therapies have achieved a more favorable response rate, time-to-progression, and/or overall survival in advanced or metastatic breast cancer patients than capecitabine monotherapy (29). Collectively, capecitabine is expected to be effective for BLBC and HER2-positive breast cancers expressing EGFR and CK5/6 in combination with chemotherapy and trastuzumab.

In conclusion, the expression of TP was higher in BLBC, and capecitabine-based chemotherapy is a likely candidate for the treatment of patients with BLBC. The results warrant clinical trials of capecitabine in combination with chemotherapy or trastuzumab based on hormonal/HER2 receptor status.

\section{References}

1. Goldhirsch A, Ingle JN, Gelber RD, Coates AS, Thurlimann B, Senn HJ and Panel Members: Thresholds for therapies: highlights of the St. Gallen International Expert Consensus on the primary therapy of early breast cancer 22009. Ann Oncol 20: 1319-1329, 2009.

2. Early Breast Cancer Trialists Collaborative Group (EBCTCG): Effects of chemotherapy and hormonal therapy for early breast cancer on recurrence and 15-year survival: an overview of the randomised trials. Lancet 365: 1687-1717, 2005.

3. Sørlie T, Perou CM, Tibshirani R, et al: Gene expression patterns of breast carcinomas distinguish tumor subclasses with clinical implications. Proc Natl Acad Sci USA 98: 10869-10874, 2001.

4. Van't Veer LJ, Dai H, van de Vijver MJ, et al: Gene expression profiling predicts clinical outcome of breast cancer. Nature 415: 530-536, 2002.

5. Perou CM, Sørlie T, Eisen MB, et al: Molecular portraits of human breast tumours. Nature 406: 747-752, 2000.

6. Carey LA, Perou CM, Livasy CA, et al: Race, breast cancer subtypes and survival in the Carolina Breast Cancer Study. JAMA 295: 2492-2502, 2006.

7. Rakha EA, El-Sayed ME, Green AR, et al: Prognostic markers in triple-negative breast cancer. Cancer 109: 25-32, 2007.

8. Blum JL: Xeloda in the treatment of metastatic breast cancer. Oncology 57 (Suppl 1): 16-20, 1999.

9. Miwa M, Ura M, Nishida M, et al: Design of a novel oral fluoropyrimidine carbamate, capecitabine, which generates 5-fluorouracil selectively in tumours by enzymes concentrated in human liver and cancer tissue. Eur J Cancer 34: 1274-1281, 1998. 
10. Fiedkin M and Roberts D: The enzymatic synthesis of nucleosides. I. Thymidine phosphorylase in mammalian tissue. J Biol Chem 207: 245-256, 1954.

11. Moghaddam A and Bicknell R: Expression of platelet-derived endothelial cell growth factor in Escherichia coli and confirmation of its thymidine phosphorylase activity. Biochemistry 31 : 12141-12146, 1992.

12. Toi M, Atiqur Rahman M, Bando H, et al: Thymidine phosphorylase (platelet-derived endothelial-cell growth factor) in cancer biology and treatment. Lancet Oncol 6: 158-166, 2005.

13. Ishikawa T, Sekiguchi F, Fukase Y, et al: Positive correlation between the efficacy of capecitabine and doxifluridine and the ration of thymidine phosphorylase to dihydropyrimidine dehydrogenase activities in tumors in human cancer xenografts. Cancer Res 58: 685-690, 1998.

14. Sawada Y, Fujii T, Takahashi H, et al: A case of triple negative chest wall recurrent breast cancer treated with capecitabine and docetaxel combination therapy (XT therapy). Gan To Kagaku Ryoho 36: 815-817, 2009.

15. Hachisuka Y, Kamei Y, Umeoka T, et al: A case of triple negative recurrent breast cancer successfully treated with capecitabine + docetaxel combination chemotherapy. Gan To Kagaku Ryoho 35: 475-458, 2008.

16. Umemura S, Shirane M, Takekoshi S, et al: Overexpression of E2F-5 correlates with a pathological basal phenotype and a worse clinical outcome. Br J Cancer 100: 764-771, 2009.

17. Kono T, Nishida M, Inagaki N, et al: Development and characterization of 1C6-203, a new monoclonal antibody specific to human thymidine phosphorylase. J Histochem Cytochem 49: 131-138, 2001.

18. Tsuda H, Akiyama F, Kurosumi M, et al: Reproducible immunohistochemical criteria based on multiple raters' judgments for expression of thymidine phosphorylase in breast cancer tissue. Breast Cancer Res Treat 86: 215-223, 2004.

19. Nielsen TO, Hsu FD, Jensen K, et al: Immunohistochemical and clinical characterization of the basal-like subtype of invasive breast carcinoma. Clin Cancer Res 10: 5367-5374, 2004.
20. Baselga J, Albanell J and Ruiz A: Phase II and tumor pharmacodynamic study of gefitinib in patients with advanced breast cancer. J Clin Oncol 23: 5323-5333, 2005.

21. Shiu KK, Tan DS and Reis-Filho JS: Development of therapeutic approaches to 'triple negative' phenotype breast cancer. Expert Opin Ther Targets 12: 1123-1137, 2008.

22. Kim JB, Stein R and O'Hare MJ: Tumour-stromal interactions in breast cancer: the role of stroma in tumourigenesis. Tumour Biol 26: 173-185, 2005.

23. Finak G, Bertos N, Pepin F, et al: Stromal gene expression predicts clinical outcome in breast cancer. Nat Med 14: 518-527, 2008.

24. Evrard A, Cuq P, Ciccolini J, et al: Increased cytotoxicity and bystander effect of 5-fluorouracil and 5-deoxy-5-fluorouridine in human colorectal cancer cells transfected with thymidine phosphorylase. Br J Cancer 80: 1726-1733, 1999.

25. Kato Y, Matsukawa S, Muraoka R, et al: Enhancement of drug sensitivity and a bystander effect in PC-9 cells transfected with a platelet-derived endothelial cell growth factor thymidine phosphorylase cDNA. Br J Cancer 75: 506-511, 1997.

26. Eda H, Fujimoto K, Watanabe S, et al: Cytokines induce thymidine phosphorylase expression in tumor cells and make them more susceptible to 5'-deoxy-5-fluorouridine. Cancer Chemother Pharmacol 32: 333-338, 1993.

27. Tripathy D: Capecitabine in combination with novel targeted agents in the management of metastatic breast cancer: underlying rationale and results of clinical trials. Oncologist 12: 375-389, 2007.

28. Fujimoto-Ouchi K, Sekiguchi F and Tanaka Y: Antitumor activity of combinations of anti-HER-2 antibody trastuzumab and oral fluoropyrimidines capecitabine/5'-dFUrd in human breast cancer models. Cancer Chemother Pharmacol 49: 211-216, 2002.

29. Mori K, Hasegawa M, Nishida M, et al: Expression levels of thymidine phosphorylase and dihydropyrimidine dehydrogenase in various human tumor tissues. Int J Oncol 17: 33-38, 2000. 